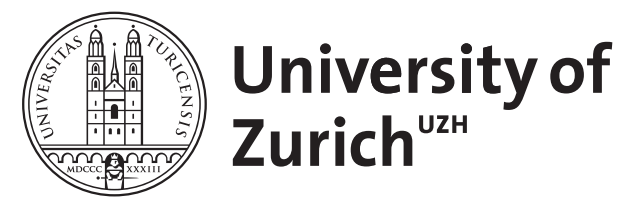

\title{
Hämoplasmeninfektion bei einer Milchkuh
}

\author{
Baggenstos, R ; Wenzinger, B ; Meli, Marina L ; Hofmann-Lehmann, Regina ; Knubben-Schweizer, \\ Gabriela
}

\begin{abstract}
The present work describes the clinical and laboratory examination as well as the treatment of a 7-year-old local dairy breed cow presented with reduced appetite, decreasing milk yield and striking yellowish discoloured skin and mucosa. The laboratory examination revealed a high degree regenerative anaemia and hyperbilirubinaemia. The bovine haemotrophic mycoplasma species Mycoplasma wenyonii and 'Candidatus Mycoplasma haemobos' were detected in the blood by PCR. Treatment with oxytetracycline rapidly improved the general condition, and milk production was increased. In a follow-up study, blood samples of all 23 animals from the same herd were examined. Fifteen cows were found to be infected with both haemoplasma species, three animals were only infected with 'Candidatus Mycoplasma haemobos' and one animal only with Mycoplasma wenyonii. Two out of three tested calves were positive for 'Candidatus Mycoplasma haemobos'. Except for the above described anaemic cow, all other animals were clinically healthy.
\end{abstract}

Other titles: Haemoplasma infection in a dairy cow

Posted at the Zurich Open Repository and Archive, University of Zurich ZORA URL: https://doi.org/10.5167/uzh-69252

Journal Article

Originally published at:

Baggenstos, R; Wenzinger, B; Meli, Marina L; Hofmann-Lehmann, Regina; Knubben-Schweizer, Gabriela (2012). Hämoplasmeninfektion bei einer Milchkuh. Tierärztliche Praxis. Ausgabe G, Grosstiere/Nutztiere, 40(6):397-400. 


\title{
Hämoplasmeninfektion bei einer Milchkuh
}

\author{
R. Baggenstos ${ }^{1}$; B. Wenzinger ${ }^{1} ;$ M. L. Meli²; R. Hofmann-Lehmann²; G. Knubben-Schweizer ${ }^{1}$
}

${ }^{1}$ Klinik für Wiederkäuer, Abteilung für Ambulanz und Bestandesmedizin der Vetsuisse-Fakultät, Universität Zürich, Schweiz; ${ }^{2}$ Veterinärmedizinisches

Labor der Vetsuisse-Fakultät, Universität Zürich, Schweiz

\section{Schlüsselwörter}

Rind, Anämie, Ikterus, Mycoplasma wenyonii, ,Candidatus Mycoplasma haemobos'

\section{Zusammenfassung}

Die Arbeit beschreibt die klinische und labordiagnostische Untersuchung und Behandlung einer 7 Jahre alten Rotfleckviehkuh mit reduzierter Fresslust und Milchleistung sowie auffällig blassgelb verfärbter Haut und Schleimhaut. Die labordiagnostische Untersuchung ergab eine hochgradige regenerative Anämie und eine Hyperbilirubinämie. Mittels PCR ließen sich die Hämoplasmenarten Mycoplasma wenyonii und ,Candidatus Mycoplasma haemobos' im Blut nachweisen. Unter der Therapie mit Oxytetrazyklin verbesserten sich der Allgemeinzustand und die Milchleistung der Kuh schnell. Bei der Untersuchung der Blutproben aller 23 Tiere im Bestand konnten bei 15 Kühen beide Hämoplasmenarten, bei drei nur ,Candidatus Mycoplasma haemobos' und bei einer Kuh nur Mycoplasma wenyonii nachgewiesen werden. Von den drei getesteten Kälbern erwiesen sich zwei als positiv für ,Candidatus Mycoplasma haemobos'. Mit Ausnahme der oben erwähnten Kuh waren alle Tiere klinisch gesund.
Key words

Cattle, anaemia, icterus, Mycoplasma wenyonii, ,Candidatus Mycoplasma haemobos'

\section{Summary}

The present work describes the clinical and laboratory examination as well as the treatment of a 7-year-old local dairy breed cow presented with reduced appetite, decreasing milk yield and striking yellowish discoloured skin and mucosa. The laboratory examination revealed a high degree regenerative anaemia and hyperbilirubinaemia. The bovine haemotrophic mycoplasma species Mycoplasma wenyonii and 'Candidatus Mycoplasma haemobos' were detected in the blood by PCR. Treatment with oxytetracycline rapidly improved the general condition, and milk production was increased. In a follow-up study, blood samples of all 23 animals from the same herd were examined. Fifteen cows were found to be infected with both haemoplasma species, three animals were only infected with 'Candidatus Mycoplasma haemobos' and one animal only with Mycoplasma wenyonii. Two out of three tested calves were positive for 'Candidatus Mycoplasma haemobos'. Except for the above described anaemic cow, all other animals were clinically healthy.

Haemoplasma infection in a dairy cow

Tierärztl Prax 2012; 40 (G): 397-400

Eingegangen: 23. März 2012

Akzeptiert nach Revision: 24. Mai 2012

\section{Departement für Nutztiere}

Abteilung Ambulanz und Bestandesmedizin

Vetsuisse-Fakultät Universität Zürich

Winterthurerstrasse 260

CH-8057 Zürich

E-Mail: rbaggenstos@vetclinics.uzh.ch

\section{Einleitung}

Hämoplasmen oder hämotrophe Mykoplasmen sind gramnegative, zellwandfreie, auf den Erythrozyten haftende Bakterien. Sie wurden weltweit bei verschiedenen Säugetieren beschrieben. Beim Rind werden die beiden Hämoplasmenarten Mycoplasma wenyonii und ,Candidatus Mycoplasma haemobos' in der Literatur erwähnt. Selten führen Infektionen mit diesen Mykoplasmen zu klinischen Symptomen wie Fieber, Anämie, Ikterus, Lymphadenopathie, Ödembildung an Hintergliedmaßen und Skrotum, Schwellungen an den Zitzen, sinkender Milchleistung, Gewichtsverlust und Fertilitätsstörungen.

\section{Fallbeschreibung}

\section{Anamnese}

Die 7 Jahre alte, im zweiten Monat trächtige Rotfleckviehkuh stammte aus einem Schweizer Milchviehbetrieb mit 20 Kühen in Anbindehaltung und drei Mastkälbern. Das Tier wurde in der ambulatorischen Klinik der Universität Zürich im Mai 2011 vorgestellt, da es seit 3 Tagen eine intermittierend reduzierte Fresslust zeigte und die Milchleistung sank.

\section{Klinische und labordiagnostische Untersuchungen}

Die Kuh zeigte einen leichtgradig reduzierten Allgemeinzustand, eine verminderte Fresslust sowie einen mäßigen Ernährungszustand. Die Körpertemperatur betrug $39^{\circ} \mathrm{C}$. Der Hautturgor und 
die Bulbi waren ohne besonderen Befund. Haarkleid und Haut präsentierten sich unauffällig, Ektoparasiten konnten nicht festgestellt werden. Die Schleimhäute waren anämisch-ikterisch, die kapilläre Füllungszeit lag bei 2 Sekunden. Die Auskultation ergab eine erhöhte Herzfrequenz (100 Schläge pro Minute), eine verstärkte Intensität des Herzschlags sowie unauffällige Befunde an der Lunge. Die Atemfrequenz lag mit 20 Zügen pro Minute im Referenzbereich. Die palpablen Lymphknoten waren ohne besonderen Befund. Mit Ausnahme einer leichtgradig reduzierten Pansenfüllung und teilweise positiven Fremdkörperproben lieferte die Untersuchung des Gastrointestinaltrakts, ebenso wie die vaginale und rektale Untersuchung keine abnormen Befunde. Der Harn war dunkelgelb verfärbt, die Untersuchung mit dem Teststreifen (Combur ${ }^{9}$ Test $^{\oplus}$, Roche, Schweiz) ergab aber unauffällige Befunde. Die Harndichte wurde nicht untersucht. Am Euter konnte eine leichte Gelbfärbung der Euterhaut festgestellt werden. Die Palpation des Euters, die Milchbeschaffenheit und auch der Schalm-Test waren unauffällig. Die Untersuchung der Gliedmaßen und Klauen sowie das Vorführen der Kuh lieferten keine Hinweise auf eine Erkrankung. Die Reaktionszeit im Glutaltest war auf 3 Minuten verkürzt. Für weiterführende Untersuchungen im Labor wurden eine EDTA-antikoagulierte Blutprobe, eine Serumblutprobe und eine Kotprobe gewonnen.

Die Blutuntersuchung (Sysmex XT-2000iv, Sysmex, Schweiz) ergab eine hochgradige Anämie mit einem Hämatokrit von 13\% (Referenzbereich [RB] [15] 25-33\%). Auch die Hämoglobinkonzentration (1,99 mmol/l, RB [15] 5,4-7,3 mmol/l) und die Erythrozytenzahl $\left(1,08 \times 10^{6} / \mu \mathrm{l}, \mathrm{RB}[15] 4,9-6,9 \times 10^{6} / \mu \mathrm{l}\right)$ unterschritten den Referenzbereich deutlich. Mikroskopisch zeigten die Erythrozyten eine geringgradige Anisozytose, eine geringgradige Poikilozytose, eine Polychromasie sowie eine mittelgradige basophile Tüpfelung. Die Leukozytenzahl und das Differenzialblutbild entsprachen dem Referenzbereich, nur die Normoblastenzahl war mit 77/100 Leukozyten erhöht (15). Bei der blutchemischen Untersuchung (Cobas Integra 800, Roche Diagnostics, Schweiz) ließen sich für folgende Parameter erhöhte Werte feststellen: Bilirubin (56,4 $\mu \mathrm{mol} / \mathrm{l}, \mathrm{RB}$ [15] 1,5-2,9 $\mu \mathrm{mol} / \mathrm{l})$, Harnstoff (6,8 mmol/l, RB [15] 2,4-6,5 mmol/l), Aspartat-Aminotransferase (AST, $178 \mathrm{U} / \mathrm{l}$, RB [15] 57-103 U/l), $\gamma$-Glutamyltransferase ( $\gamma$-GT, 46 U/l, RB [15] 13-32 U/l) und Glutamatdehydrogenase (GLDH, 134,5 U/l, RB [15] 4-18,2 U/1). Die Kreatinkinaseaktivität lag im Referenzbereich (15). Mit Ausnahme einer leicht erniedrigten Phosphatkonzentration (1,19 mmol/l, RB [15] 1,3-2,4 mmol/l) befanden sich die Elektrolytwerte innerhalb der Referenzbereiche (15).

Die mikroskopische Untersuchung eines Blutausstrichs auf Blutparasiten verlief negativ. Die Kotuntersuchung auf Fasciola hepatica lieferte ebenfalls einen negativen Befund und bei der Begehung der Weiden konnten keine Feuchtstellen und somit allfällige Habitate für den Zwischenwirt Galba truncatula von Fasciola hepatica entdeckt werden.

\section{Diagnose und Behandlung}

Die klinischen und hämatologischen Befunde waren vereinbar mit einer Anämie. Die erhöhte Normoblastenzahl sprach für eine regenerative Anämie. Da die Plasmaproteinkonzentration mit 5,1 mmol/l (RB [15] 3,9-5,3 mmol/l) im Referenzbereich lag, konnte eine hämorrhagische Anämie weitgehend ausgeschlossen werden. Es deutete alles auf eine hämolytische Anämie hin. Die Harnuntersuchung ergab keine Hämoglobinurie, was für eine extravasale Hämolyse sprach. Infolgedessen wurde eine Infektion mit Anaplasma-Spezies vermutet, eine entsprechende PCR-Untersuchung des Blutes veranlasst und die Kuh für 3 Tage mit Oxytetrazyklin in doppelter Dosierung (10 mg/kg KM, Engemycin ${ }^{\circledR} 10 \%$ ad us. vet., Veterinaria AG, Schweiz) behandelt (16). Da die PCRUntersuchung auf Anaplasma-Spezies (A. marginale, A. phagocytophilum, A. spp.) negativ verlief, erfolgte eine Untersuchung auf Theileria spp., Babesia spp., Rickettsia helvetica, Rickettsia spp. und hämotrophe Mykoplasmen. Alle PCR-Tests erbrachten negative Befunde mit Ausnahme der Hämoplasmen-PCR, die bezüglich Mycoplasma wenyonii und ,Candidatus Mycoplasma haemobos' positiv war.

\section{Weiterer Verlauf}

Die Kuh erholte sich unter der Therapie sehr schnell. Sie zeigte nach 2 Tagen wieder eine ungestörte Futteraufnahme und die Milchleistung stieg wieder an. Knapp 3 Wochen nach der Diagnosestellung fand eine Untersuchung auf Bestandsebene statt. Die 20 Milchkühe und drei Mastkälber im Alter von 2 Monaten präsentierten sich klinisch unauffällig. Die mit EDTA antikoagulierten Blutproben aller Tiere wurden direkt nach der Entnahme im Labor mittels PCR auf Mycoplasma wenyonii und, Candidatus Mycoplasma haemobos' untersucht. Fünfzehn der 20 Kühe erwiesen sich als positiv für beide Hämoplasmenarten, zwei waren positiv für ,Candidatus Mycoplasma haemobos', eine Kuh war positiv für Mycoplasma wenyonii und bei einer Kuh lieferte die Untersuchung einen negativen Befund für beide Hämoplasmenarten. Die klinisch auffällige Kuh war zu diesem Zeitpunkt negativ bezüglich Mycoplasma wenyonii, aber nach wie vor positiv für, Candidatus Mycoplasma haemobos'. Zwei der drei Kälber waren positiv für ,Candidatus Mycoplasma haemobos', bei dem dritten Kalb wurde für beide Hämoplasmenarten ein negativer Befund festgestellt. Somit waren 69,6\% aller Tiere im Bestand positiv für Mycoplasma wenyonii und 87,0\% der Tiere positiv für, Candidatus Mycoplasma haemobos'.

Der Hämatokrit lag bei 12 von 20 Kühen mit 26-34\% in oder knapp über dem Referenzbereich (RB [15] 25-33\%). Bei sieben Kühen bestand ein erniedrigter Hämatokrit mit Werten zwischen 22 und 24\%. Der Hämatokrit der klinisch erkrankten Kuh betrug zu diesem Zeitpunkt 16\%. Der Hämatokrit der drei Kälber lag zwischen 32 und $38 \%$. 


\section{Diskussion}

Hämotrophe Mykoplasmen oder Hämoplasmen gehören zu den gramnegativen, zellwandfreien, auf den Erythrozyten haftenden Bakterien (10). Hämoplasmen wurden weltweit bei verschiedenen Säugetieren beschrieben (8). Ehemals der Ordnung Rickettsien, Familie Anaplasmataceae, Genera Eperythrozoon und Haemobartonella zugeteilt, gehören sie seit 2001, basierend auf der 16SrRNA-Gensequenz, zur Klasse Mollicutes, zum Genus Mycoplasma (hämotrophe Mykoplasmen, Hämoplasmen) (10, 11).

Beim Rind werden Mycoplasma wenyonii und ,Candidatus Mycoplasma haemobos' in der Literatur erwähnt. Einen ersten Fallbericht veröffentlichten Adler und Ellenbogen im Jahr 1934 (1). Eine Kasuistik aus der Veterinärmedizinischen Fakultät in Auburn 1994 beschrieb die Infektion eines Charolais-Stieres mit Mycoplasma wenyonii. Die auffälligsten Symptome neben einer ausgeprägten Anämie und Fieber waren eine Ödembildung am Skrotum und an den Hintergliedmaßen. Ein Ikterus konnte bei diesem Tier nicht festgestellt werden (9). Ein ähnlicher Fallbericht über eine Kuh mit einer Infektion durch Mycoplasma wenyonii wurde vor kurzem durch die Oklahoma State University veröffentlicht (2).

Es existieren auch Fallberichte, bei denen mehrere Tiere einer Herde an Hämoplasmen erkrankt sind. Smith et al. (13) dokumentierten 1990 den Ausbruch einer Infektion mit Mycoplasma wenyonii in einer Milchviehherde. Zehn von 100 frisch abgekalbten Rindern wiesen Schwellungen an den Zitzen und den Hintergliedmaßen auf. Die Kniefaltenlymphknoten waren vergrößert und die Tiere zeigten Fieber. Die Milchleistung sank, die Rinder verloren an Gewicht und es kam zu Fruchtbarkeitsstörungen. Die klinischen Symptome gingen mit einer hohen Anzahl an Mycoplasma wenyonii im Blutausstrich einher und verschwanden mit der Reduktion oder dem völligen Verschwinden der Erreger aus dem Blut (13). Hoelzle et al. (3) beschrieben die klinischen Symptome Anämie, sinkende Milchleistung, Infertilität und Lahmheit bei drei infizierten Herden.

Ferner wird über den Nachweis von Mycoplasma wenyonii und ,Candidatus Mycoplasma haemobos' im Zusammenhang mit Anaplasmoseausbrüchen berichtet $(4,6)$. Hofmann-Lehmann et al. (4) untersuchten 2004 in der Schweiz eine Milchkuhherde von knapp 300 Tieren, die Schwäche, Appetitverlust, Milchleistungsabfall, Fieber, eine hämolytische Anämie und Hämoglobinurie zeigten. Auslöser der Erkrankung war Anaplasma marginale. Bei einigen Tieren ließen sich aber auch Babesia spp., Theileria spp., Anaplasma phagocytophilum, Mycoplasma wenyonii und, zum ersten Mal in der Schweiz, ,Candidatus Mycoplasma haemobos' im Blut nachweisen (4). Ein ähnlicher Bericht erschien später in Ungarn (5). Auch hier konnten bei einem Anaplasmoseausbruch Mycoplasma wenyonii und ,Candidatus Mycoplasma haemobos' gefunden werden. In beiden Fällen wird jedoch vermutet, dass die Hämoplasmen nicht die Hauptursache der klinischen Symptome waren. Allerdings ist bekannt, dass hämotrophe Bakterien den pathogenen Effekt von Anaplasma marginale verstärken können (6).

In einer Untersuchung aus Japan aus dem Jahr 2008 waren 34 von 78 wegen einer anderen Krankheit an eine Klinik eingewiese- ne Kühe für eine oder beide der Hämoplasmenarten positiv (14). Da in der Schweiz ähnliche Ergebnisse auch bei gesunden Rindern gefunden wurden, ist nicht davon auszugehen, dass eine andere Grunderkrankung die Infektion mit bovinen Hämoplasmen begünstigen muss (7).

Bei der Übertragung spielen vermutlich hauptsächlich blutsaugende Arthropoden eine Rolle. Hofmann-Lehmann et al. (4) untersuchten im Zusammenhang mit dem Anaplasmoseausbruch 2002 in der Schweiz Läuse, Zecken und Fliegen, die von den erkrankten Tieren gesammelt wurden, mittels PCR auf Mycoplasma wenyonii. In allen untersuchten Läusen und Fliegen (je fünf Stück), aber in keiner der sechs untersuchten Zecken war Mycoplasma wenyonii nachweisbar. Hornok et al. (6) erzielten 2011 bei ihren Untersuchungen in Ungarn ähnliche Ergebnisse und auch eine transplazentare Übertragung der bovinen Hämoplasmen wurde nachgewiesen.

Die klinische Bedeutung der bovinen Hämoplasmen ist bis dato nicht eindeutig geklärt. Im untersuchten Betrieb konnten keine vermehrt auftretenden Erkrankungen oder Bestandsprobleme wie Fertilitätsstörungen festgestellt werden. Die im vorliegenden Fallbericht beschriebene Kuh wies eine extravasale hämolytische Anämie und als Folge davon einen Ikterus auf. Warum gerade diese Kuh aus dem Bestand klinische Symptome zeigte, ist unklar. Es ist nicht auszuschließen, dass bei ihr zum Zeitpunkt der Untersuchung eine andere Erkrankung vorlag. Aufgrund der positiven Fremdkörperproben und der verkürzten Reaktionszeit im Glutaltest lag der Verdacht auf eine Fremdkörpererkrankung nahe. Diese konnte auch dadurch nicht ganz ausgeschlossen werden, dass sich der Allgemeinzustand der Kuh unter der Oxytetrazyklintherapie verbesserte, während der Hämatokrit von 13\% auf 16\% anstieg. In der Folgeuntersuchung knapp 3 Wochen nach der 3-tägigen Therapie ließ sich $M y$ koplasma wenyonii nicht mehr nachweisen. Die Kuh war aber nach wie vor positiv für, Candidatus Mycoplasma haemobos'. Ob sich diese Hämoplasmen in der Anzahl reduziert hatten, war nicht festzustellen. Dies ist jedoch denkbar, da Smith et al. (13) zeigen konnten, dass die klinischen Symptome bereits mit einer Reduktion der Hämoplasmenzahl verschwanden (13).

Wie häufig Hämoplasmen bei Rindern auf Bestandsebene in der Schweiz vorkommen und wie hoch deren Bedeutung einzuschätzen ist, ist Gegenstand weiterer Untersuchungen.

\section{Interessenkonflikt}

Die Autoren bestätigen, dass kein Interessenkonflikt besteht.

\section{Fazit für die Praxis}

Klinische Symptome bei Rindern aufgrund einer Infektion mit bovinen Hämoplasmen werden nur selten beschrieben. Kommt es zu einer Störung des Allgemeinbefindens mit Symptomen wie Fieber, Anämie, Ikterus, geschwollenen Lymphknoten oder Ödembildung, sind Infektionen mit bovinen Hämoplasmen in die Liste der Differenzialdiagnosen aufzunehmen. In einem Verdachtsfall empfiehlt sich eine Therapie mit Oxytetrazyklin. 


\section{Literatur}

1. Adler S, Ellenbogen V. A note on two new blood parasites of cattle, Eperythrozoon and Bartonella. J Comp Path Ther 1934; 47: 219-221.

2. Genova SG, Streeter RN, Velguth KE, Snider TA, Kocan KM, Simpson KM. Severe anemia associated with Mycoplasma wenyonii infection in a mature cow. Can Vet J 2011; 52: 1018-1021.

3. Hoelzle K, Winkler M, Kramer MM, Wittenbrink MM, Dieckmann SM, Hoelzle LE. Detection of Candidatus Mycoplasma haemobos in cattle with anaemia. Vet J 2010; 187: 408-410.

4. Hofmann-Lehmann R, Meli ML, Dreher UM, Gonczi E, Deplazes P, Braun U, Engels M, Schüpbach J, Jörger K, Thoma R, Griot C, Stärk KD, Willi B Schmid J, Kocan KM, Lutz H. Concurrent infections with vectore-borne pathogens associated with fatal hemolytic anemia in a cattle herd in Switzerland. J Clin Microbiol 2004; 42: 3775-3780.

5. Hornok S, Micusutka A, Fernández de Mera IG, Meli ML, Gönczi E, Tánczos B, Mangold AJ, Farkas R, Lutz H, Hofmann-Lehmann R, de la Fuente J. Fatal bovine anaplasmosis in a herd with new genotypes of Anaplasma marginale, Anaplasma ovis and concurrent haemoplasmosis. Res Vet Sci 2012; 92: 30-35.

6. Hornok S, Micsutka A, Meli ML, Lutz H, Hofmann-Lehmann R. Molecular investigation of transplacental and vector-borne transmission of bovine haemoplasmas. Vet Microbiol 2011; 152: 411-414.

7. Meli ML, Willi B, Dreher UM, Cattori V, Knubben-Schweizer G, Nuss K, Braun U, Lutz H, Hofmann-Lehmann R. Identification, molecular characterization, and occurrence of two bovine hemoplasma species in Swiss cattle and development of real-time TaqMan quantitative PCR assays for diagnosis of bovine hemoplasma infections. J Clin Microbiol 2010; 48: 3563-3568.

8. Messick JB. Hemotrophic mycoplasmas (hemoplasmas): a review and new insights into pathogenic potential. Vet Clin Pathol 2004; 33: 2-13.

9. Montes AJ, Wolfe DF, Welles EG, Tyler JW, Tepe E. Infertility associated with Eperythrozoon wenyonii infection in a bull. J Am Vet Med Assoc 1994; 204 : 261-263.

10. Neimark H, Johansson KE, Rikihisa Y, Tully JG. Proposal to transfer some members of the genera Haemobartonella and Eperythrozoon to the genus Mycoplasma with descriptions of 'Candidatus Mycoplasma haemofelis', 'Candidatus Mycoplasma haemomuris', 'Candidatus Mycoplasma haemosuis' and 'Candidatus Mycoplasma wenyonii'. Int J Syst Evol Microbiol 2001; 51: 891-899.

11. Neimark H, Johansson KE, Rikihisa Y, Tully JG. Revision of haemotrophic Mycoplasma species names. Int J Syst Evol Microbiol 2002; 52: 683.

12. Radostits OM, Gay CC, Hinchcliff KW, Constable PD. Diseases associated with Rickettsiales. In: Veterinary Medicine. A Textbook of the Diseases of Cattle, Horses, Sheep, Pigs, and Goats. Philadelphia: Saunders Elsevier 2007; 1458 .

13. Smith JA, Thrall MA, Smith JL, Salman MD, Ching SV, Collins JK. Eperythrozoon wenyonii infection in dairy cattle. J Am Vet Med Assoc 1990; 196: 1244-1250.

14. Tagawa M, Matsumotot K, Inokuma H. Molecular detection of Mycoplasma wenyonii and 'Candidatus Mycoplasma haemobos' in cattle in Hokkaido, Japan Vet Microbiol 2008; 132: 177-180.

15. Veterinärmedizinisches Labor, Departement für Nutztiere, Vetsuisse-Fakultät Zürich. Laboreigene Referenzwerte.

\section{Meldung \\ VETIDATA}

VETIDATA stellt eine Informationsplattform zu Fragen in Bezug auf Arzneimittelanwendung, Toxikologie und Arzneimittelrecht dar. Umfassende Informationen wie aktuelle Rechtsvorschriften, Angaben zu Präparaten, Impfstoffen, Wirkstoffen und zur Rückstandsproblematik erhalten Sie über den Internetauftritt unter http://www.vetidata.de oder die bundeseinheitliche Servicerufnummer. Per Telefon, Fax oder E-Mail können auch individuelle Fragestellungen geklärt werden.
Der Zugriff auf die Webseite ist registrierten Nutzern vorbehalten. Zur Registrierung gelangen Sie mit den allgemeinen Zugangsdaten:

Benutzername: praxis
Kennwort: forum

Das Entgelt für den Zugriff per Internet beträgt derzeit 55,- $€ / J a h r$ zzgl. der geltenden Mehrwertsteuer. Innerhalb einer Testphase von vier Wochen nach der erstmaligen Registrierung können Nutzer den Vertrag kündigen, ohne dass weitere finanzielle Forderungen entstehen.

\section{VETIDATA}

Veterinärmedizinischer Informationsdienst für Arzneimittelanwendung, Toxikologie und Arzneimittelrecht An den Tierkliniken 15 04103 Leipzig http://www.vetidata.de E-Mail: info@vetidata.de

Fax: 03 41-97 38149 Servicerufnummer für Anfragen: 01 80-500 9119 (0,14 €/Minute im Festnetz, max. 0,42 €/Minute aus Mobilfunknetzen), Montag-Freitag: 9:00-16:00 Uhr 